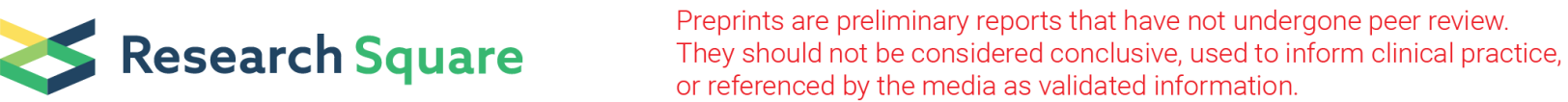 \\ Vesicle shape transformations driven by confined active filaments
}

\section{Matthew Peterson}

Brandeis University https://orcid.org/0000-0002-5749-7770

\section{Aparna Baskaran}

Brandeis University

\section{Michael Hagan ( $\sim$ hagan@brandeis.edu )}

Brandeis University https://orcid.org/0000-0002-9211-2434

\section{Article}

Keywords: Active Matter Systems, Deformable Boundaries, Particle-based Simulations, Active Stress Organization, Emergent Behaviors, Targeted Shape Dynamics

Posted Date: May 6th, 2021

DOl: https://doi.org/10.21203/rs.3.rs-448564/v1

License: (c) (i) This work is licensed under a Creative Commons Attribution 4.0 International License. Read Full License

Version of Record: A version of this preprint was published at Nature Communications on December 1st, 2021. See the published version at https://doi.org/10.1038/s41467-021-27310-8. 


\title{
Vesicle shape transformations driven by confined active filaments
}

\author{
Matthew S. E. Peterson, Aparna Baskaran, ${ }^{*}$ and Michael F. Hagan ${ }^{\dagger}$ \\ Martin A. Fisher School of Physics, Brandeis University, Waltham, MA, 02453
}

(Dated: April 21, 2021)

\begin{abstract}
In active matter systems, deformable boundaries provide a mechanism to organize internal active stresses and perform work on the external environment. To study a minimal model of such a system, we perform particle-based simulations of an elastic vesicle containing a collection of polar active filaments. The interplay between the active stress organization due to interparticle interactions and that due to the deformability of the confinement leads to a variety of filament spatiotemporal organizations that have not been observed in bulk systems or under rigid confinement, including highly-aligned rings and caps. In turn, these filament assemblies drive dramatic and tunable transformations of the vesicle shape and its dynamics. We present simple scaling models that reveal the mechanisms underlying these emergent behaviors and yield design principles for engineering active materials with targeted shape dynamics.
\end{abstract}

\section{INTRODUCTION}

Active matter encompasses systems whose microscopic constituents consume energy at the particle scale to produce forces and motion. Novel macroscale phenomena emerge in these systems when these forces collectively organize into mesoscale 'active stresses'. Harnessing these active stresses to drive particular emergent behaviors could enable a new class of materials with life-like properties that would be impossible in traditional equilibrium materials. However, rationally designing active constituents to generate a desired emergent behavior requires identifying the principles that govern organization of mesoscopic active stresses. Similarly, many biological functions, such as cytoplasmic streaming, morphogenesis, and cell migration, are driven by active stresses that emerge from active components confined within a cell [1-8]. Understanding physical mechanisms that underlie these functions is a key goal of cellular biophysics.

As a step toward these goals, this article describes a computational and theoretical study of a minimal model of active filaments enclosed within a deformable vesicle. We thereby identify a generic route to control selforganizing active stresses by enclosing active components with anisotropic shapes and/or internal degrees of freedom within deformable confining boundaries. Our results demonstrate that coupling between boundary deformations and the assembly of internal active components leads to a positive feedback capable of driving diverse nonlinear transformations of morphology and dynamics.

The field of active matter has identified two key mechanisms that control self-organization of active stresses: (1) anisotropic interactions between active components that realign forces, and (2) confining boundaries. For example, interactions between self-propelled particles that drive interparticle alignment result in bands or flocks [9, 10], changing the length and stiffness of active polymers leads

\footnotetext{
*aparna@brandeis.edu

$\dagger$ hagan@brandeis.edu
}

to dramatic reorganization of active stresses [11, 12], and confining active particles leads to system-spanning effects such as spontaneous flow [13-20]. Furthermore, deformable confining boundaries enable non-equilibrium boundary fluctuations [21-26], including elongated tendrils and bolas [22]. The latter results suggest that flexibility is a key characteristic of a confining boundary, as it allows shape transformations, sensing and response to environmental cues, and performing work on the surroundings. Achieving such capabilities is critical to leverage minimal bio-derived experimental systems (e.g. [23, 27, 28]) to engineer controlled shape transformations. However, little is known about the behaviors that may arise when these two active stress organization modes are combined. In particular, most existing theoretical and computational studies have focused on rigid boundaries [29-32], isotropic active particles $[21,22,24,33-36]$, or have been in $2 \mathrm{D}$ [3739].

In this work, we use Langevin dynamics simulations of polar self-propelled semiflexible filaments confined within $3 \mathrm{D}$ flexible vesicles to study the combination of active interparticle alignment interactions and 3D deformable confinement. Our simulations show that interplay between these two organization modes leads to a positive feedback, in which active forces drive boundary deformation while passive stresses from the boundary guide and reinforce self-organization of the internal active stresses. This leads to a rich variety of steady-state behaviors that have not been observed in bulk systems or under rigid confinement, including highly-aligned rings, and caps that have tunable self-limited sizes, number, and symmetry. Each filament organization drives a characteristic largescale vesicle shape transformation that can be selected by varying parameters such as filament length, density, and flexibility. Asymmetric states lead to net vesicle motion, consistent with the recent experiments which find that enclosing self-propelled particles, such as bacteria, in droplets can lead to collective motility [40]. We present simple scaling analyses that reveal how the feedback between vesicle geometry and filament organization drives and stabilizes these emergent behaviors. The applicability of these scaling arguments suggests that these behaviors 
arise generically due to feedback between vesicle elasticity and active filament organization, independent of the specific model.

Understanding the fundamental mechanisms that govern this coupling between self-organization of active stress and deformations of a flexible boundary will establish design principles for soft robotics, artificial cells, or other advanced materials that mimic the capabilities of living organisms. From a biological perspective, our minimal model is not intended to directly describe the cytoskeleton - in this model, active filament propulsion enters in the overdamped limit without long-ranged hydrodynamic coupling. However, the generic feedback between vesicle elasticity and active filament organization identified in our simulations and scaling arguments may elucidate how mesoscopic active stresses in the cellular cytoskeleton drive large scale cellular shape transformations that underlie essential biological functions such as motility [1, 2], division [3-5], and endo-/exocytosis [6, 7].

\section{METHODS}

We simulate a system of $N_{\text {fil }}$ active filaments confined within an elastic vesicle, which has radius $R_{\text {ves }}$ in its undeformed state. We represent active filaments using the model in Joshi, et al. [41] - modified so that the active forcing is polar rather than nematic - in which each filament is a nearly-inextensible, semiflexible chain of $M$ beads of diameter $\sigma[42]$. Bonded beads interact through an expanded FENE potential [43], while non-bonded beads interact through a purely repulsive expanded WeeksChandler-Andersen (eWCA) potential [44] with strength $\epsilon$. The equilibrium bond length is set to $b_{\text {fil }}=\sigma / 2$ to minimize surface roughness between interacting filaments, thereby preventing filaments from interlocking at high density [45-49]. The filaments are made semiflexible with bending rigidity $\kappa_{\text {fil }}$ through a harmonic angle potential applied to each set of three consecutive beads along the chain. Since our model is not intended to describe any specific biofilament system, we incorporate activity in a minimal manner - a polar active force of magnitude $f_{\mathrm{a}}$ acts on each bead, in a direction tangent to the filament and toward the filament head. The filament volume fraction in the undeformed state is given by $\phi=N_{\mathrm{fil}} V_{\mathrm{fil}} / V_{\mathrm{ves}}$, where $V_{\text {fil }}=\pi \sigma^{3} / 6+(M-1) \pi b \sigma^{2} / 4$ is the approximate volume of a single filament - accounting for the overlap of bonded monomers - and $V_{\text {ves }}=4 \pi R_{\text {ves }}^{3} / 3$ is the nominal volume of the vesicle. Since the mesh topology is conserved in our simulations, we model an elastic vesicle. In subsequent work we plan to consider the effects of fluidizing the vesicle and imposing area or volume constraints.

We simulate the coupled Langevin equations for the filament and vesicle bead dynamics using LAMMPS [50], modified to include the active force. We neglect longranged hydrodynamic interactions for this system of high filament density; we will investigate their effect in a future study. We have set units such that the mass of all beads is

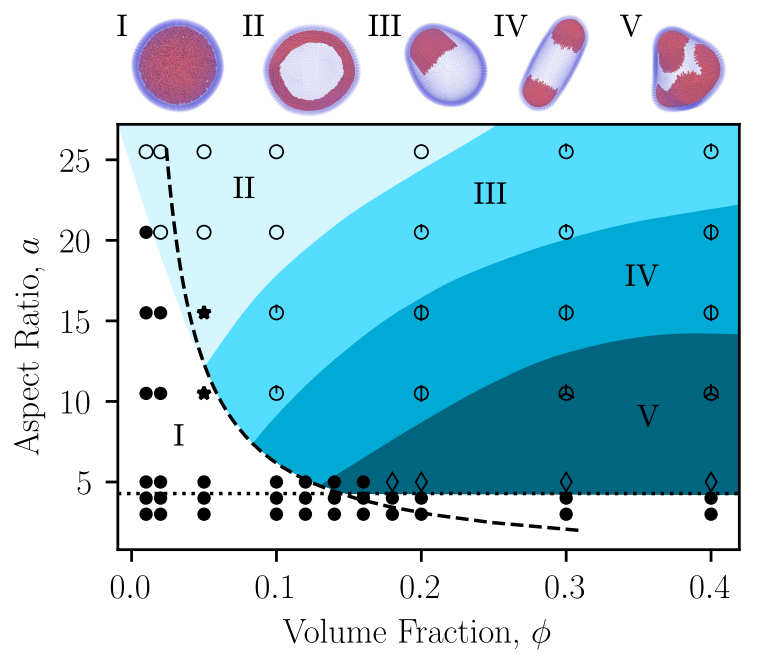

FIG. 1. Snapshots illustrating steady-state configurations of the vesicle and enclosed active filaments as a function of filament aspect ratio $a$ and initial volume fraction $\phi$. See SI Movie 1 for animations of the corresponding simulations [51]. The marked regions of parameter space indicate the typical vesicle conformation: (I) spherical, (II) oblate, (III) polar-prolate, $(I V)$ apolar-prolate, and ( $V$ ) polyhedral. The symbols associate the conformation with the internal filament organization: homogeneous throughout the bulk or on the surface, with no vesicle deformation $(\bullet)$; transient clusters and/or bands, with oblate vesicle shapes $(\star)$; stable polar rings $(0)$; stable caps (o, with a number of intersecting lines equal to the median number of caps); and dynamic caps $(\diamond)$. The dashed line shows the transition to aligned states predicted from the competition between the characteristic collision and reorientation timescales $\left(\phi=(\pi / 4)^{2} / a\right)$ described in the text, and the horizontal dotted line indicates the approximate threshold aspect ratio for the filaments to be in the strong confinement limit. Other parameters are filament bending modulus $\kappa_{\mathrm{fil}}=10^{4}$ and vesicle radius $R_{\mathrm{ves}}=25$.

$m=1$, and energies, lengths, and time are respectively in units of $k_{\mathrm{B}} T, \sigma$, and $\tau=\sqrt{m \sigma^{2} / \epsilon}$. The friction constant is set to $\gamma=1 / \tau$. For additional model details, see the Supplemental Materials [51].

\section{RESULTS AND DISCUSSION}

\section{A. Simulation results}

To discover the steady-state conformations that arise due to coupling between active propulsion and elasticity, we have performed simulations over a wide range of control parameters (Fig. 1, Fig. 3, Fig. 5, and Fig. 6): the volume fraction of filaments in the vesicle $\phi \in[0.01,0.4]$, filament aspect ratio $a=1+(M-1)\left(b_{\text {fil }} / \sigma\right) \in[3,25.5]$, active propulsion strength $f_{\mathrm{a}} \in[0,10]$, filament stiffness $\kappa_{\mathrm{fil}} \in$ $\left[10^{2}, 10^{4}\right]$, and vesicle rigidity $\kappa_{\mathrm{ves}} \in\left[10^{2}, 10^{4}\right]$.

Fig. 1 shows the steady-states as a function of filament volume fraction and aspect ratio for moderate activity $f_{\mathrm{a}}=8$. At this activity and vesicle size, for aspect 
a)

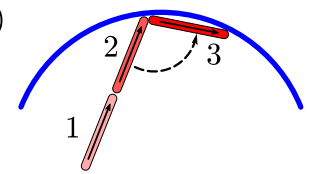

b)

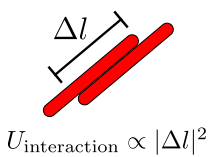

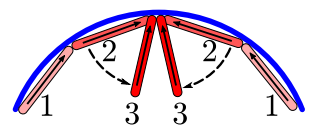

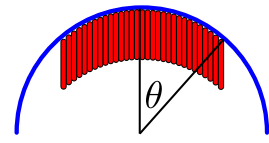

FIG. 2. a) The onset of ring and cap formation is determined by a competition of timescales: the timescale associated with rotations parallel to the vesicle (left) and the timescale associated with collisions that tend to orient the filaments perpendicular to the vesicle (right). b) Schematic of the theory for the number of caps (Eqs. (1) and (2)). We assume an activityinduced effective attractive interaction that is quadratic in the rod-rod contact length $\Delta l$ (left). The cap is assumed circular, with size parameterized by the angle $\theta$ between the cap center and edge. Vesicle curvature leads to a shearing of rods within the cap (right).

ratios $a \gtrsim\left(8 \pi R_{\text {ves }} / f_{\mathrm{a}}\right)^{1 / 3} \approx 4.3$ the system is in the strong confinement limit: because the persistence length $l_{\mathrm{p}}^{\mathrm{COM}} \propto f_{\mathrm{a}} a^{3}$ of the filament center-of-mass motion is larger than the vesicle size $l_{\mathrm{p}}^{\mathrm{COM}}>2 R_{\mathrm{ves}}$, most filaments are found on the vesicle surface at all times [52] (see SI Sec. A [51]).

Under these conditions we can classify the steady-state vesicle conformations into several categories: (I) spherical, (II) oblate, (III) polar-prolate, (IV) apolar-prolate, and $(V)$ polyhedral. These vesicle configurations are tightly coupled to the spatiotemporal organization of the filaments within, as follows.

(I): Spherical vesicle shapes arise at low filament volume fractions and aspect ratios. Under these conditions, filament-filament collisions are rare and inter-filament aligning forces are weak [53-59]. Thus, filament positions and orientations are homogeneous (throughout the vesicle interior below strong confinement, or on the vesicle surface above strong confinement), leading to little deformation of the vesicle.

(II): For low volume fraction but high aspect ratios, such that the filament length $L=a \sigma$ is comparable to the unperturbed vesicle radius, $L \sim R_{\text {ves }}$, the vesicle deforms into oblate spheroid conformations. This transition is driven by the filaments organizing into a stable polar band, which deforms the vesicle along a geodesic. This filament arrangement closely resembles the polar bands observed on the surface of rigid spheres for active particles with polar propulsion and polar interparticle alignment interactions [35], which arise due to topological requirements for a surface-constrained polarization field [31]. However, note that such polar bands would be unstable in our system if the confining geometry was a rigid sphere because the filament-filament interactions in our system are nematic (head-tail symmetric) [60, 61]. The finite deformability of the vesicle is essential to stabilize this

configuration - active forces due to the polar band force the vesicle into an oblate shape, which in turn provides a restoring force to stabilize filament alignment within the band. In support of this conclusion, simulations on infinitely rigid vesicles did not exhibit stable polar bands (see Fig. 6b and SI movie 6). Thus, this configuration provides a concrete example of how feedback between passive stresses and self-organization of active stresses can generate steady states that would be otherwise disallowed by symmetry.

$(I I I-I V)$ : For intermediate volume fractions and aspect ratios, the vesicle deforms into a prolate spheroid. These prolate vesicle conformations can be further classified by their motion, either polar (III) or apolar (IV). Further increasing the volume fraction or decreasing the aspect ratio leads to polyhedral conformations, $(V)$. States (III$V$ ) all result from filaments assembling into crystalline caps in which the rods are highly aligned and perpendicular to the vesicle surface. Interestingly, the caps are 'selflimited' in that their typical size decreases with decreasing aspect ratio, but is roughly independent of the total number of filaments $N_{\text {fil }}$ in the vesicle. Increasing $N_{\text {fil }}$ at fixed aspect ratio increases the number of caps; we observe up to 12 caps for the finite vesicle size that we consider (Fig. 4). Further, caps drive local curvature of the vesicle, leading to elasticity-mediated cap-cap repulsions which favor symmetric arrangements of caps. Thus, the vesicle morphology can be sensitively tuned by controlling filament aspect ratio and density to achieve a specific number of caps. The polar-prolate (III), apolar-prolate $(I V)$, and polyhedral states $(V)$ respectively have 1,2 , and $\geq 3$ caps. Generally, states with two or more caps do not exhibit directed motion. However, for enough caps in the vesicle (typically more than 3 ), the caps themselves can become motile, and collide with, merge with, and split from other caps (see below).

\section{B. Mechanisms underlying stress organization and deformation}

To understand how these conformations are governed by the interplay between propulsion-induced aligning forces, vesicle deformability, and vesicle curvature, we develop simple scaling estimates for the timescales and forces that govern filament alignment and interactions with the vesicle. First, we consider the transition between undeformed spherical vesicle states characterized by unaligned or weakly aligned filaments as in state (I), to the highly deformed oblate, prolate, and polyhedral vesicle shapes of states $(I I-V)$. Our simulations demonstrate that such significant vesicle shape deformations occur when filamentfilament interactions mediate the organization of ordered structures either in the plane of the vesicle or orthogonal to it.

a. The onset of filament assembly: The onset of this transition can be understood by considering a competition between two characteristic timescales that respec- 


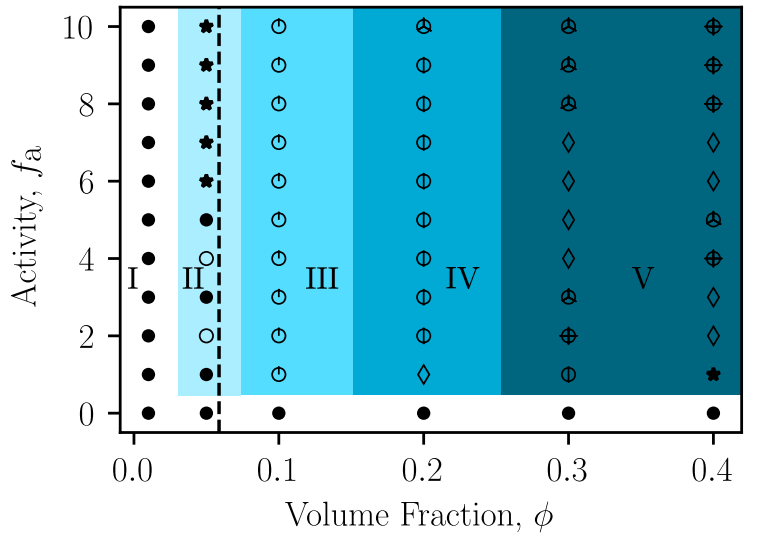

FIG. 3. Steady-state configurations as a function of $\phi$ and active force $f_{\mathrm{a}}$. The marked regions are defined as in Fig. 1 . The dashed line shows the transition to aligned states predicted by the timescale competition, which is independent of $f_{\mathrm{a}}$. Other parameters are $a=10.5$ and $\kappa_{\mathrm{fil}}=10^{4}$. See SI Movie 2 for corresponding animations.

tively govern collision-induced filament-vesicle alignment and filament-filament alignment (see Fig. 2). Filamentvesicle collisions, which tend to reorient filaments parallel to the surface $[62,63]$, have a characteristic timescale $\tau_{\text {rot }} \sim L / v_{0}$ [64], with $v_{0}=f_{\mathrm{a}} / \gamma$ the filament selfpropulsion velocity. We can estimate the timescale for filament interactions by considering filament-filament pairwise collisions whose timescale is given by $\tau_{\text {coll }} \sim \sigma / v_{0} \phi$ (see SI [51]). Thus, deformed vesicle states will arise when $\tau_{\text {coll }}<\tau_{\text {rot }}$ or equivalently $a \phi>c$, where $c \cong(\pi / 4)^{2}$ is independent of activity and filament length (see [51]). This defines a boundary separating highly deformed states of the vesicle from the undeformed spherical states (the dashed line in Fig. 1).

Notably, the active force drops out of this argument because both collision and reorientation times are $\propto f_{\mathrm{a}}$. Thus, the theory predicts that the emergence of deformed vesicle states is independent of activity of the enclosed filaments (above a threshold activity). As a test of this prediction, Fig. 3 shows the steady-states as a function of $\phi$ and $f_{\mathrm{a}}$ for fixed aspect ratio $a=10.5$. Indeed, formation of large deformations does not depend on activity, with non-spherical shapes forming for $\phi \geq c / a \approx 0.06$ (as predicted by the above timescale argument) for all $f_{\mathrm{a}}>0$ that we considered.

This simple theoretical picture gives a predictive principle, in terms of properties of the active filaments, for when vesicle shape transformations occur. However, the theory assumes the strong activity, long filament limit and thus neglects thermal noise. Below a threshold activity $\left(f_{\mathrm{a}} \lesssim 1\right.$ in our units) the vesicle will not deform because filament organization is destroyed by thermal fluctuations. Also, cap formation (and thus vesicle shape transitions) do not occur when the filaments are below the strong confinement limit discussed above $(a \lesssim 4.3$ for the parameters of Fig. 1, shown as a dotted line).

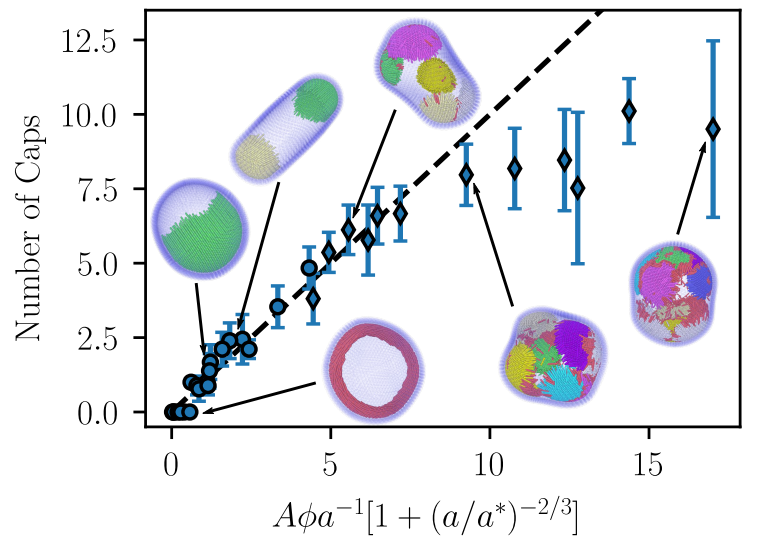

FIG. 4. The number of caps measured in simulations (symbols) compared to the theory (Eqs. (1) and (2), dashed line), with $A=4 R_{\mathrm{ves}} / \sqrt{3} \pi \sigma$ and $a^{*} \approx 130$ (chosen by eye). Diamonds indicate dynamic cap states. Note that the number of caps in the simulation results is likely under counted for the dynamic states due to the caps' motility. The simulation data is the same as in Fig. 1. Active filaments are colored by which cap they belong to for visual clarity.

b. Cap morphologies: We can derive further insight into shape transformations by considering the system in the strongly deformed regime with polyhedral shapes. The defining characteristic underlying these states is filament assembly into well-ordered caps. Most cap states are relatively static, with occasional association/dissociation of individual rods (See SI Movie 4), except for the parameters that lead to the highly dynamic, reconfiguring caps discussed below. In a static steady state, the active and elastic forces must balance. In particular, the dense crystalline nature of caps arises because the active force and the presence of the vesicle surface leads to an effective attractive interaction between nearby filaments. This attraction drives radial growth of a cap, since filaments on the cap periphery have fewer neighbors, leading to an effective interfacial tension. This effect is both reinforced by and competes with vesicle elasticity. The active force of small caps drives vesicle deformations whose local curvature enhances effective filament-filament attractions. However, as the cap grows in radius, vesicle curvature drives an effective shear of filaments (see Fig. 2b) that reduces rod-rod overlaps and thus opposes the active force.

We describe this competition by constructing an effective 'free energy' whose gradients correspond to the active and passive forces (Fig. 2b). Since the active force favors rods to align in a smectic layer, the shear due to vesicle curvature imposes an 'energy' cost of $U_{\text {shear }}(\theta)=n_{\text {cap }} 2 \pi R_{\text {ves }}^{2} \frac{G}{2}[\cos \theta+\sec \theta-2]$, with $\theta$ the angle subtended by the cap on the vesicle surface, $n_{\text {cap }}$ the number of caps, and a 'shear modulus' $G \sim f_{\mathrm{a}}$ (but independent of $L_{\mathrm{rod}}$ ) [51]. In the strongly deformed region the caps are roughly circular, so the interfacial energy is given by $U_{\text {int }}(\theta)=n_{\text {cap }} 2 \pi R_{\text {ves }} \gamma \sin \theta$, with the 'interfacial tension' $\lambda \sim L_{\text {rod }} f_{\text {a }}$ accounting for the diminished inter- 
actions at the cap boundary. This results in a free energy as a function of cap size [51]:

$$
f(\theta)=\frac{1}{1-\cos \theta}\left[\frac{1}{2}(\cos \theta+\sec \theta-2)+\zeta \sin \theta\right]
$$

where $\zeta=G / \gamma R_{\text {ves }} \sim L / R_{\text {ves }}$ is given by the balance between the effective interfacial tension and shear modulus, and should be linear in filament length but roughly independent of $f_{\mathrm{a}}$ since both of these effects are driven by activity.

Minimizing this per-filament free energy yields an optimal $\theta[65,66]$ corresponding to the self-limited cap size. Assuming that we are well above the onset of cap formation so that essentially all filaments are in caps,

$$
n_{\text {cap }} \propto \phi a^{-1}\left[1+\left(a / a^{*}\right)^{-2 / 3}\right]
$$

where $a^{*} \propto R_{\mathrm{ves}} / \sigma$ is an adjustable parameter that may depend on activity. This expression holds provided $a \ll$ $a^{*}$. For the data in Fig. 1, we obtain $a^{*} \approx 130$, leading to the dashed line shown in Fig. 4.

Except for states with many $\left(n_{\text {cap }} \gtrsim 7\right)$ motile caps, there is close agreement between the observed and predicted $n_{\text {cap }}$. Above this threshold our cap-counting algorithm likely under counts $n_{\text {cap }}$, since different caps are often adjacent and interacting. Further, the prediction of Eqs. (1) and (2) that the self-limited cap size is independent of activity is consistent with observations at different $f_{\mathrm{a}}$ (see Fig. 3). The motile cap states appear to arise when the curved vesicle geometry forces interactions between the inward-facing ends of adjacent caps. Such interactions occur above a threshold number and aspect ratio of filaments, given by $N_{\text {fil }} \gtrsim C\left(1-a \sigma / R_{\text {ves }}\right)^{2}$, where $C$ is a constant (see SI Sec. D [51]).

We note that the geometric factors governing the selflimited cap size parallel those in a recently studied equilibrium system of rigid filaments end-adsorbed onto a rigid spherical nanoparticle, which self-assemble due to direct pairwise inter-filament attractions [66]. However, in the present system, the effective filament-filament interactions and vesicle geometry are many-body and emergent in that they arise due to feedback between non-equilibrium active forces and vesicle deformations.

\section{Effect of filament and vesicle rigidity}

Thus far, we have focused on the interplay between activity and vesicle deformability by performing simulations in the limit of rigid rods, $\kappa_{\mathrm{fil}}=10^{4}$, and high (but finite) vesicle rigidity $\kappa_{\mathrm{ves}}=5 \times 10^{3}$. We now briefly discuss the effect of allowing for finite filament and vesicle flexibility.

Fig. 5 shows the vesicle conformation and filament organization states as a function of filament bending modulus and activity, for fixed filament volume fraction $\phi=0.2$. We see that for finite filament flexibility, the transition to aligned ring and cap states is suppressed

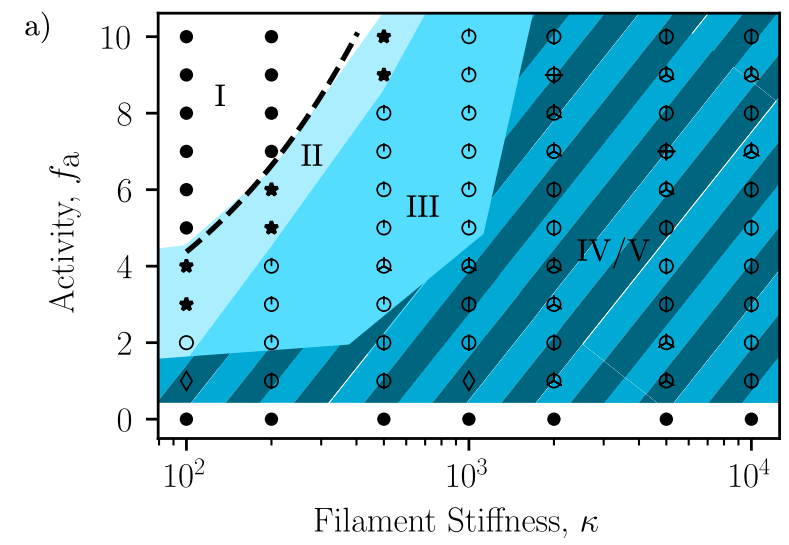

b)

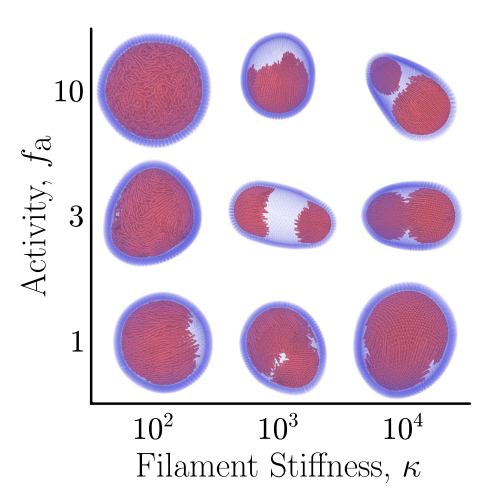

FIG. 5. a) Vesicle conformations and filament organizations as a function of filament rigidity $\kappa_{\text {fil }}$ and active force strength $f_{\mathrm{a}}$, for volume fraction $\phi=0.2$ and filament aspect ratio $a=10.5$. For a given filament stiffness, increasing activity reduces the number of caps until an upper-threshold activity value $f_{\mathrm{a}}^{\mathrm{SC}}$, beyond which the system transitions into an undeformed state. As described in SI section E, this transition occurs because activity renormalizes the filament bending modulus to smaller values[41], thus reducing filament alignment interactions and causing the system to leave the strong confinement limit. The dashed line shows the prediction for $f_{\mathrm{a}}^{\mathrm{SC}}$ given by Eq. S35. Note that there is no adjustable parameter. In the rigid rod limit $\left(\kappa_{\mathrm{fil}}>10^{3}\right)$ all non-zero active force values that we simulated led to cap formation. b) Selected snapshots of states shown in (a). Animations of these states can be found in SI Movie 3.

above a threshold activity, which decreases with decreasing $\kappa_{\text {fil }}$.

This result can be understood as follows. On generic grounds, decreasing the filament rigidity will reduce the tendency for filaments to align and thus impede the formation of aligned rings and caps. For filament stiffness values well below the rigid rod limit, the process by which caps and rings form is more complicated than considered previously. The upper-threshold activity for filament organization can be, at least in part, explained by the observation that activity renormalizes filament rigidity to smaller values according to $\kappa_{\text {fil }}^{\text {eff }} \cong \kappa_{\text {fil }} /\left(1+f_{\mathrm{a}}^{2}\right)[41]$. Interactions between flexible active agents is such that the active energy preferentially dissipates into bend modes, 


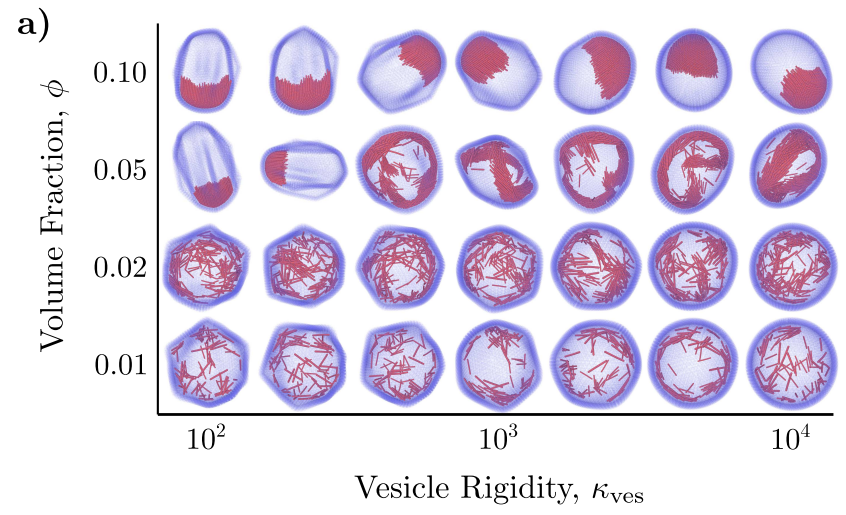

b)

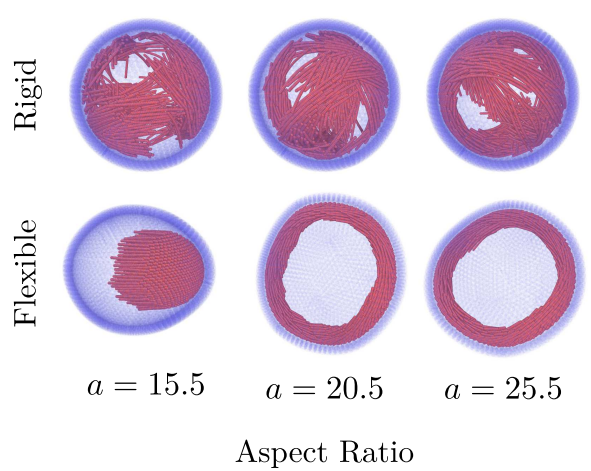

FIG. 6. a) Simulation snapshots illustrating vesicle conformations and filament organizations as a function of filament volume fraction and vesicle rigidity. As the vesicle rigidity is reduced below a critical value (corresponding to the critical Föppl-von Kármán number FvK $\approx 154$ [67]), the vesicle undergoes a buckling transition leading to the formation of facets. While we observe most of the same classes of filament selforganization in faceted and round vesicles, polar bands trace a dynamic path between vertices in faceted vesicles, while they trace a stable geodesic in round vesicles. Animations of these states can be found in SI movie 5. b) Comparisons between flexible and rigid vesicles as a function of filament aspect ratio, with other parameters set to $f_{\mathrm{a}}=8, \phi=0.1$, and $\kappa_{\mathrm{fil}}=10^{4}$. In contrast to flexible vesicles, rigid vesicles do not allow for the formation of stable caps or rings. When polar rings do form in rigid vesicles, they are transient - continuously breaking and reforming over the course of the trajectory. Animations of this comparison can be found in SI movie 6 .

effectively increasing filament flexibility and therefore suppressing filament alignment. In particular, the upperthreshold activity corresponds to the point when the activity-renormalized flexibility of filaments causes the system to leave the strong confinement limit. This occurs for $f_{\mathrm{a}} \gtrsim C \kappa_{\mathrm{fil}}^{3 / 5}$, where $C=\left(8 \pi R_{\text {ves }}\right)^{-1 / 5}$ (see SI Sec. D for details), which is shown as the dashed line in Fig. 5 .

Fig. $6 \mathrm{a}$ shows the conformations obtained by varying the vesicle rigidity $\kappa_{\text {ves }}$ and filament volume fraction $\phi$, while fixing the filament rigidity $\kappa_{\mathrm{fil}}=10^{4}$ and active force $f_{\mathrm{a}}=8$. The most striking effect of reducing the vesicle rigidity is that it drives a faceting transition when the the Föppl-von Kármán number, $\mathrm{FvK}=Y R_{\text {ves }}^{2} / \kappa$ where $Y$ is the Young's modulus of the vesicle and $\kappa=\sqrt{3} \kappa_{\text {ves }}$ is the bending modulus [68], is increased above a critical value, $\mathrm{FvK} \gtrsim 154$. This is an equilibrium property of an elastic vesicle, independent of the active filaments [67]. Our results indicate that faceting does not qualitatively change the formation of caps, but that caps form at slightly lower filament volume fraction for reduced vesicle bending modulus. This could be anticipated from the theoretical arguments described above, since reducing the bending modulus allows filaments' active forces to further deform the vesicle, leading to a smaller local radius of curvature in the vicinity of a cap. More interestingly, the facets appear to destabilize the polar bands and rings. For round vesicles (with bending modulus such that FvK < 154), a stable ring forms along a geodesic. In contrast, in faceted vesicles at the same activity and filament volume fraction, rings or bands tend to form paths that connect facet vertices. The bending of the ring path imposed by the facet connectivity destabilizes the ring, causing it to transiently break and reform (similar to the transient band state described above). This behavior suggests that it will be interesting to explore the possibility of coupling between vesicle faceting and filament organization in a future work.

Fig. $6 \mathrm{~b}$ compares configurations observed with a flexible vesicle $\left(\kappa_{\mathrm{ves}}=5 \times 10^{3}\right)$ and a rigid vesicle $\left(\kappa_{\mathrm{ves}} \rightarrow \infty\right)$ for $a \in[15.5,25.5], \phi=0.10, f_{\mathrm{a}}=8$, and $\kappa_{\mathrm{fil}}=10^{4}$. While the flexible vesicle exhibits stable polar rings and single caps at these parameters (Fig. 1), the rigid vesicle system is unable to form the single-cap state, and only exhibits transient polar rings, which continuously break apart and reform as the simulation progresses. These results emphasize the importance of the feedback between active stress organization and vesicle deformation, which allows for stable states that are otherwise inaccessible under rigid confinement.

\section{CONCLUSIONS}

This work demonstrates that confining active filaments within a deformable vesicle leads to multiple transformations of the vesicle shape and motility, which can be precisely tuned by control parameters. The feedback enabled by coupling deformable boundaries with anisotropic particles significantly enriches the available modes of selforganization. While the self-limited caps are the most striking class of such behaviors, the stable polar bands for particles with nematic interactions provides a clear example of how boundary deformations can stabilize novel states. Notably, both of these classes of behaviors arise due to a spontaneous symmetry breaking of the initially spherical boundary.

These results have implications for future experiments on active materials constructed from anisotropic particles confined within deformable boundaries. In particular, the transitions can be controlled by tuning parameters that are readily accessible in experiments - filament length, 
flexibility, and volume fraction. In contrast, activity is a complicated function of motor properties and ATP in bio-derived systems [69, 70]. Thus, our computational results suggest strategies to engineer active vesicles with designable shapes and dynamics, and other capabilities resembling those of living cells. Furthermore, our theoretical analysis identifies the mechanisms that underlie these emergent morphologies by revealing how filamentfilament interactions and vesicle deformations couple to spatiotemporally organize stress. This provides a modelindependent roadmap for exploring additional classes of emergent functionalities in parameter regimes beyond the scope of the present work, including highly deformable fluidized vesicles and other symmetries of activity.

\section{ACKNOWLEDGMENTS}

We acknowledge support from NSF DMR-1855914 and the Brandeis Center for Bioinspired Soft Materials, an NSF MRSEC (DMR-2011846). We also acknowledge computational support from NSF XSEDE computing resources allocation TG-MCB090163 (Stampede and Comet) and the Brandeis HPCC which is partially supported by DMR-MRSEC 2011486. We also acknowledge the KITP Active20 program, during which some of these ideas were developed, which is supported in part by the National Science Foundation under Grant No. NSF PHY1748958.

Author contributions: MSEP, AB, and MFH designed the research; MSEP performed the computational modeling; MSEP, AB, and MFH performed the theoretical modeling; MSEP analyzed the data; and MSEP, AB, and $\mathrm{MFH}$ wrote the paper.

Competing interests: The authors declare that they have no competing interests.
[1] P. Sens, Proc. Natl. Acad. Sci. U. S. A. , 202011785 (2020).

[2] R. Ananthakrishnan and A. Ehrlicher, Int. J. Biol. Sci. , 303 (2007).

[3] M. Leptin and B. Grunewald, Development 110, 73 (1990).

[4] A. L. Miller, Curr. Biol. 21, R976 (2011).

[5] O. Polyakov, B. He, M. Swan, J. W. Shaevitz, M. Kaschube, and E. Wieschaus, Biophys. J. 107, 998 (2014).

[6] J. M. Besterman and R. B. Low, Biochem. J. 210, 1 (1983).

[7] G. J. Doherty and H. T. McMahon, Annu. Rev. Biochem. 78, 857 (2009).

[8] Y. Maroudas-Sacks, L. Garion, L. Shani-Zerbib, A. Livshits, E. Braun, and K. Keren, Nat. Phys. (2020), 10.1038/s41567-020-01083-1.

[9] V. Schaller, C. Weber, C. Semmrich, E. Frey, and A. R. Bausch, Nature 467, 73 (2010).

[10] A. Bricard, J.-B. Caussin, N. Desreumaux, O. Dauchot, and D. Bartolo, Nature 503, 95 (2013).

[11] A. Senoussi, S. Kashida, R. Voituriez, J.-C. Galas, A. Maitra, and A. Estevez-Torres, Proc. Natl. Acad. Sci. 116, 22464 (2019).

[12] T. Strübing, A. Khosravanizadeh, A. Vilfan, E. Bodenschatz, R. Golestanian, and I. Guido, Nano Letters 20, 6281 (2020).

[13] S. A. Edwards and J. M. Yeomans, Europhys. Lett. 85, 18008 (2009).

[14] F. G. Woodhouse and R. E. Goldstein, Phys. Rev. Lett. 109, 168105 (2012).

[15] H. Wioland, F. G. Woodhouse, J. Dunkel, J. O. Kessler, and R. E. Goldstein, Phys. Rev. Lett. 110, 268102 (2013).

[16] M. Ravnik and J. M. Yeomans, Phys. Rev. Lett. 110, 026001 (2013).

[17] E. Lushi, H. Wioland, and R. E. Goldstein, Proc. Natl. Acad. Sci. U. S. A. 111, 9733 (2014).

[18] H. Wioland, E. Lushi, and R. E. Goldstein, New J. Phys.
18, 075002 (2016).

[19] K.-T. Wu, J. B. Hishamunda, D. T. N. Chen, S. J. DeCamp, Y.-W. Chang, A. Fernández-Nieves, S. Fraden, and Z. Dogic, Science 355 (2017).

[20] M. Varghese, A. Baskaran, M. F. Hagan, and A. Baskaran, Phys. Rev. Lett. 125, 268003 (2020).

[21] Y. Li and P. R. ten Wolde, Phys. Rev. Lett. 123, 148003 (2019).

[22] H. R. Vutukuri, M. Hoore, C. Abaurrea-Velasco, L. van Buren, A. Dutto, T. Auth, D. A. Fedosov, G. Gompper, and J. Vermant, Nature 586, 52 (2020).

[23] F. C. Keber, E. Loiseau, T. Sanchez, S. J. DeCamp, L. Giomi, M. J. Bowick, M. C. Marchetti, Z. Dogic, and A. R. Bausch, Science 345, 1135 (2014).

[24] S. C. Takatori and A. Sahu, Phys. Rev. Lett. 124, 158102 (2020).

[25] R. Hughes and J. M. Yeomans, Phys. Rev. E 102, 020601 (2020).

[26] P. W. Miller, N. Stoop, and J. Dunkel, Phys. Rev. Lett. 120, 268001 (2018).

[27] M. Elbaum, D. K. Fygenson, and A. Libchaber, Phys. Rev. Lett. 76, 4078 (1996).

[28] D. K. Fygenson, J. F. Marko, and A. Libchaber, Phys. Rev. Lett. 79, 4497 (1997).

[29] M. M. Norton, A. Baskaran, A. Opathalage, B. Langeslay, S. Fraden, A. Baskaran, and M. F. Hagan, Phys. Rev. E 97, 012702 (2018).

[30] S. Henkes, M. C. Marchetti, and R. Sknepnek, Phys. Rev. E 97, 042605 (2018).

[31] S. Shankar, M. J. Bowick, and M. C. Marchetti, Phys. Rev. X 7, 031039 (2017).

[32] F. Alaimo, C. Köhler, and A. Voigt, Sci. Rep. 7, 5211 (2017).

[33] M. Paoluzzi, R. Di Leonardo, M. C. Marchetti, and L. Angelani, Sci. Rep. 6, 34146 (2016).

[34] C. Wang, Y.-k. Guo, W.-d. Tian, and K. Chen, J. Chem. Phys. 150, 044907 (2019). 
[35] R. Sknepnek and S. Henkes, Phys. Rev. E 91, 022306 (2015).

[36] I. R. Bruss and S. C. Glotzer, Soft matter 13, 5117 (2017).

[37] C. Abaurrea-Velasco, T. Auth, and G. Gompper, New J. Phys. 21, 123024 (2019).

[38] A. C. Quillen, J. P. Smucker, and A. Peshkov, Phys. Rev. E 101, 052618 (2020).

[39] G. A. Vliegenthart, A. Ravichandran, M. Ripoll, T. Auth, and G. Gompper, Sci. Adv. 6 (2020), 10.1126/sciadv.aaw9975.

[40] M. Rajabi, H. Baza, T. Turiv, and O. D. Lavrentovich, Nat. Phys. 17, 260 (2020).

[41] A. Joshi, E. Putzig, A. Baskaran, and M. F. Hagan, Soft Matter 15, 94 (2019).

[42] R. G. Winkler and G. Gompper, J. Chem. Phys. 153, 040901 (2020).

[43] K. Kremer and G. S. Grest, J. Chem. Phys. 92, 5057 (1990).

[44] J. D. Weeks, D. Chandler, and H. C. Andersen, J. Chem. Phys. 54, 5237 (1971).

[45] J. Elgeti and G. Gompper, Europhys. Lett. 101, 48003 (2013).

[46] R. E. Isele-Holder, J. Elgeti, and G. Gompper, Soft Matter 11, 7181 (2015).

[47] R. E. Isele-Holder, J. Jäger, G. Saggiorato, J. Elgeti, and G. Gompper, Soft Matter 12, 8495 (2016).

[48] Ö. Duman, R. E. Isele-Holder, J. Elgeti, and G. Gompper, Soft Matter 14, 4483 (2018).

[49] R. Chelakkot, M. F. Hagan, L. Mahadevan, and A. Gopinath, bioRxiv (2020), 10.1101/2020.06.08.140731.

[50] S. Plimpton, J. Comp. Phys. 117, 1 (1995).

[51] See Supplemental Material at the end of this document for model details and additional figures.

[52] Y. Fily, A. Baskaran, and M. F. Hagan, Soft Matter 10, 5609 (2014).

[53] F. Peruani, A. Deutsch, and M. Bär, Phys. Rev. E 74, 030904 (2006).

[54] F. Peruani, J. Starruß, V. Jakovljevic, L. SøgaardAndersen, A. Deutsch, and M. Bär, Phys. Rev. Lett. 108, 098102 (2012).

[55] A. Baskaran and M. C. Marchetti, Phys. Rev. Lett. 101, 268101 (2008).

[56] A. Baskaran and M. C. Marchetti, Phys. Rev. E 77, 011920 (2008).

[57] S. R. McCandlish, A. Baskaran, and M. F. Hagan, Soft Matter 8, 2527 (2012).

[58] F. Ginelli, F. Peruani, M. Bär, and H. Chaté, Phys. Rev. Lett. 104, 184502 (2010).
[59] M. Bär, R. Grossmann, S. Heidenreich, and F. Peruani, Annu. Rev. Condens. Matter Phys. 11, 441 (2020).

[60] E. Bertin, H. Chaté, F. Ginelli, S. Mishra, A. Peshkov, and S. Ramaswamy, New J. Phys. 15, 085032 (2013).

[61] S. Ngo, A. Peshkov, I. S. Aranson, E. Bertin, F. Ginelli, and H. Chaté, Phys. Rev. Lett. 113, 038302 (2014).

[62] H. H. Wensink and H. Löwen, Phys. Rev. E 78, 031409 (2008).

[63] C. Bechinger, R. Di Leonardo, H. Löwen, C. Reichhardt, G. Volpe, and G. Volpe, Rev. Mod. Phys. 88, 045006 (2016).

[64] The ability of collisions of self-propelled particles on a surface to drive formation of smectic layers is supported by a recent observation in bacterial colonies growing on flat surfaces, in which bacteria form 'rosettes' with the rod-like bacteria oriented perpendicular to the surface [71] However, in contrast to the self-limited caps in our system, the bacterial rosettes do not exhibit a preferred size because they are on a flat boundary.

[65] M. F. Hagan and G. M. Grason, "Equilibrium mechanisms of self-limiting assembly," (2021), arXiv:2007.01927 [condmat.soft].

[66] N. Yu, A. Ghosh, and M. F. Hagan, Soft Matter 12, 8990 (2016).

[67] J. Lidmar, L. Mirny, and D. R. Nelson, Phys. Rev. E 68, 051910 (2003).

[68] G. Gompper and D. M. Kroll, J. Phys. I France 6, 1305 (1996).

[69] L. M. Lemma, M. M. Norton, S. J. DeCamp, S. A. Aghvami, S. Fraden, M. F. Hagan, and Z. Dogic, arXiv:2006.15184 [cond-mat] (2020), arXiv:2006.15184 [cond-mat].

[70] P. Chandrakar, J. Berezney, B. Lemma, B. Hishamunda, A. Berry, K.-T. Wu, R. Subramanian, J. Chung, D. Needleman, J. Gelles, and Z. Dogic, "Microtubule-based active fluids with improved lifetime, temporal stability and miscibility with passive soft materials," (2018), arXiv:1811.05026 [cond-mat.soft].

[71] O. J. Meacock, A. Doostmohammadi, K. R. Foster, J. M. Yeomans, and W. M. Durham, Nat. Phys. (2020), 10.1038/s41567-020-01070-6.

[72] Y. Fily, A. Baskaran, and M. F. Hagan, "Active particles on curved surfaces," (2016), arXiv:1601.00324 [condmat.soft].

[73] M. Doi and S. F. Edwards, The Theory of Polymer Dynamics, International Series of Monographs on Physics No. 73 (Clarendon Press, Oxford, 2007). 
Figures

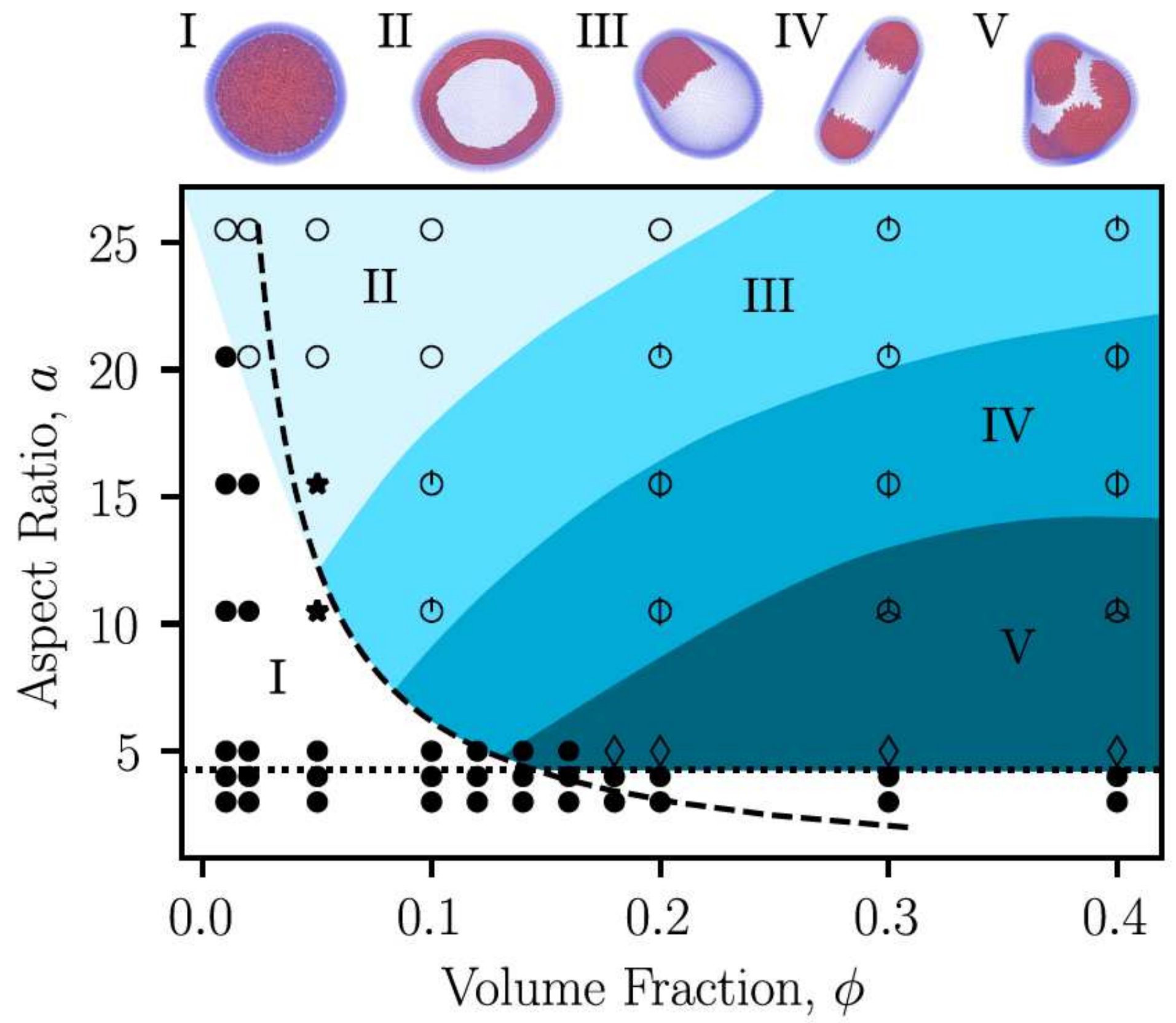

Figure 1

Please see manuscript .pdf for full caption. 
a)
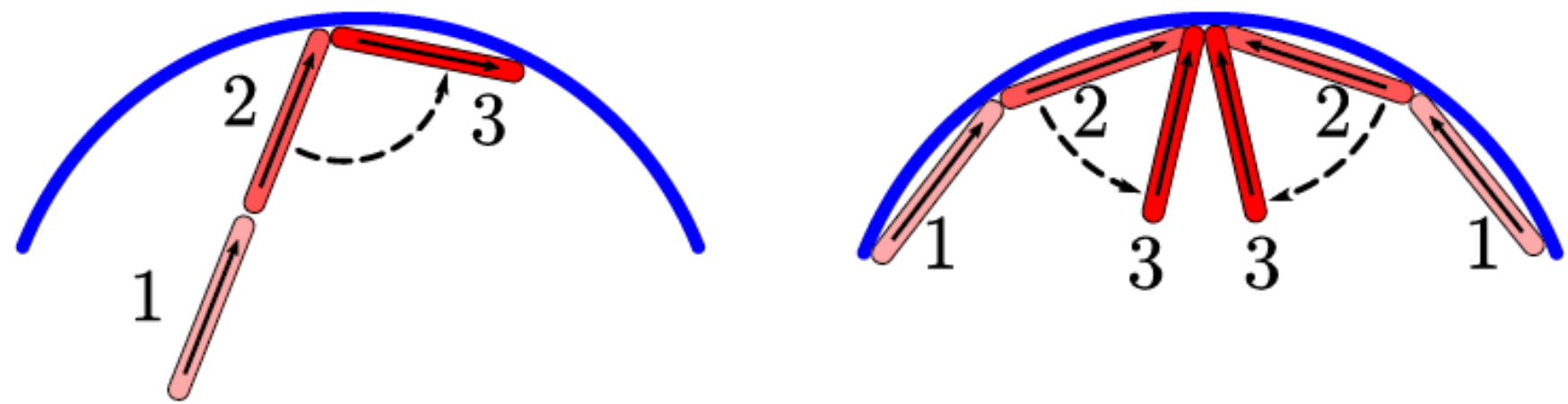

b)
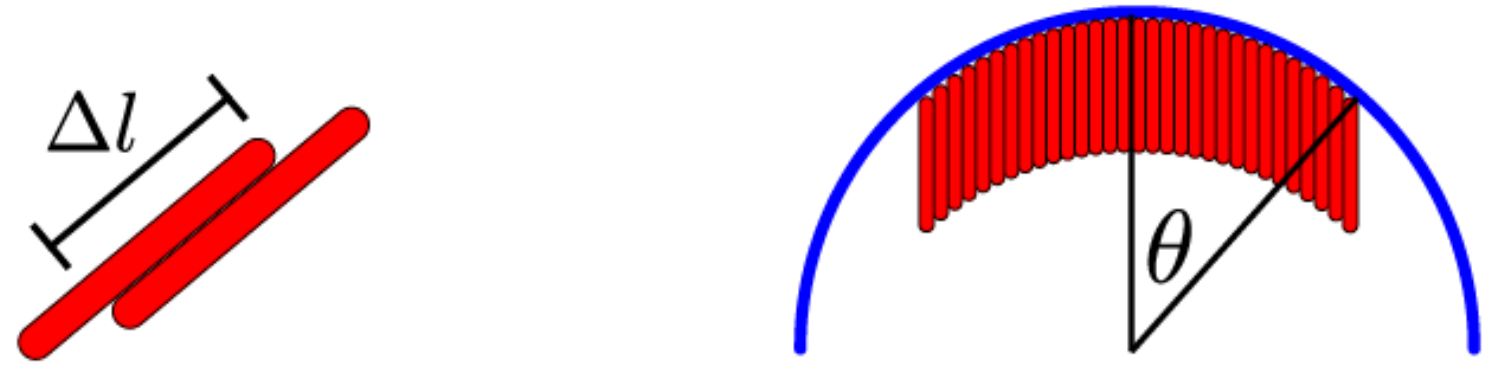

$U_{\text {interaction }} \propto|\Delta l|^{2}$

Figure 2

Please see manuscript .pdf for full caption. 


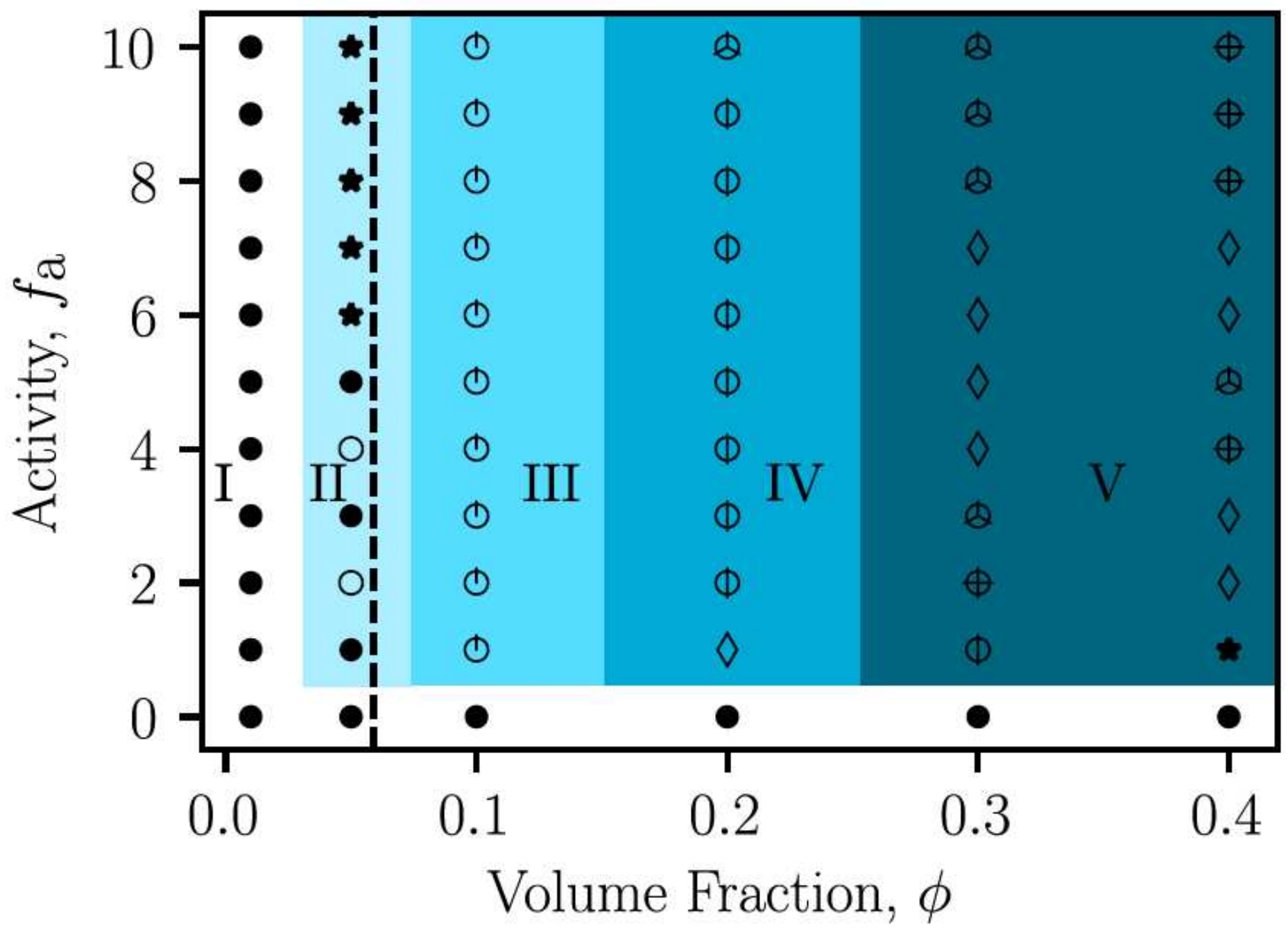

Figure 3

Please see manuscript .pdf for full caption. 


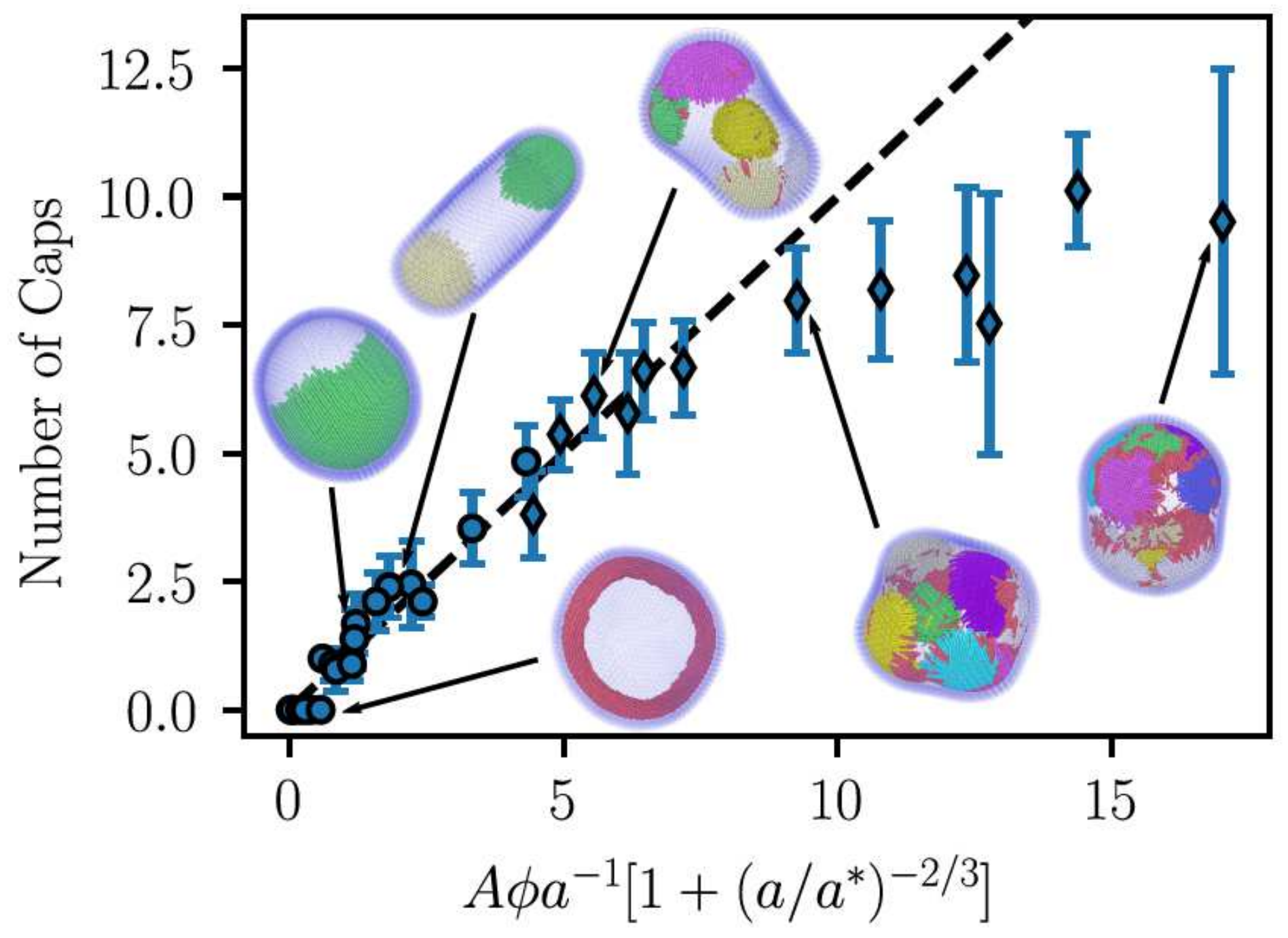

Figure 4

Please see manuscript .pdf for full caption. 
a)

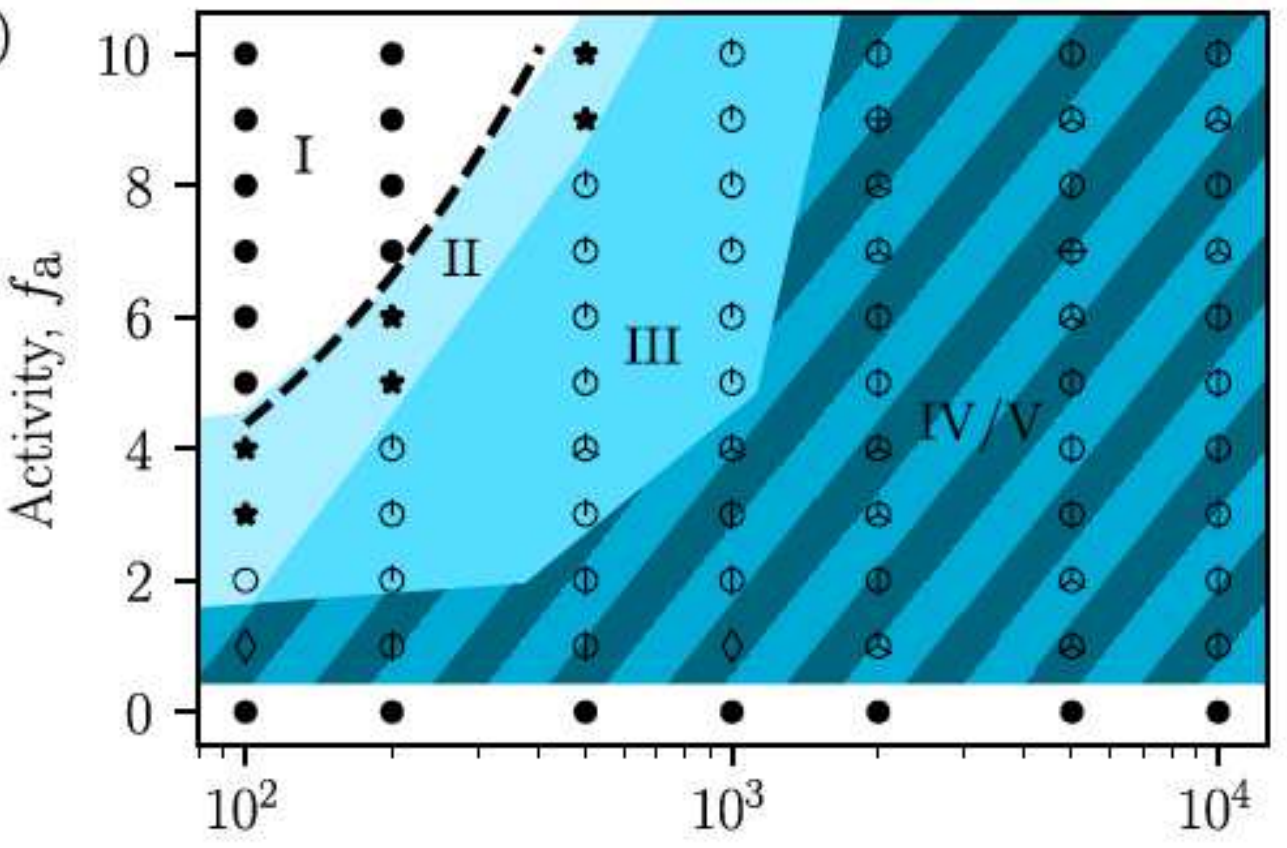

Filament Stiffness, $\kappa$

b)

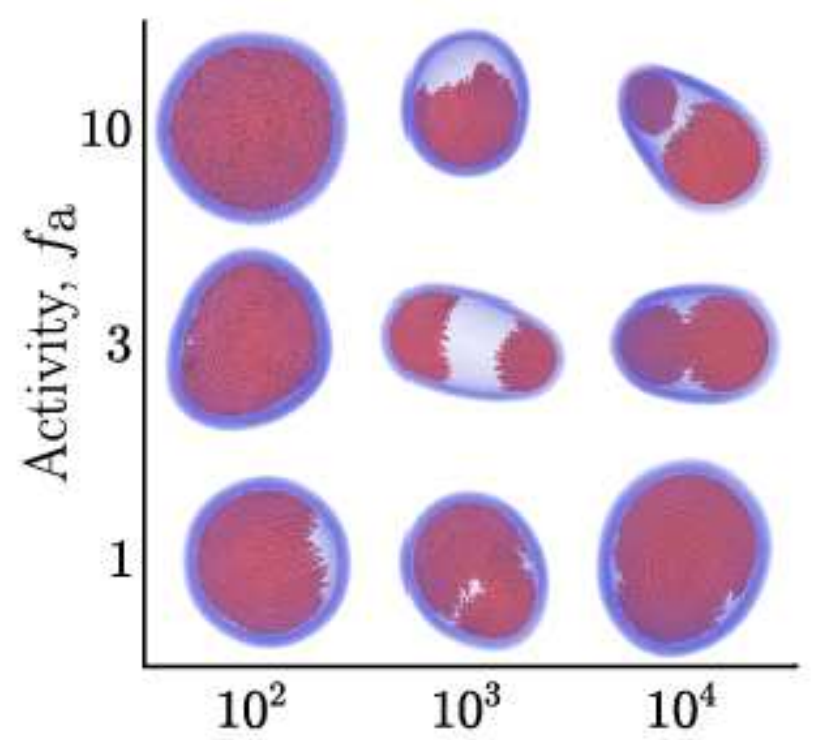

Filament Stiffness, $\kappa$

Figure 5

Please see manuscript .pdf for full caption. 
a)

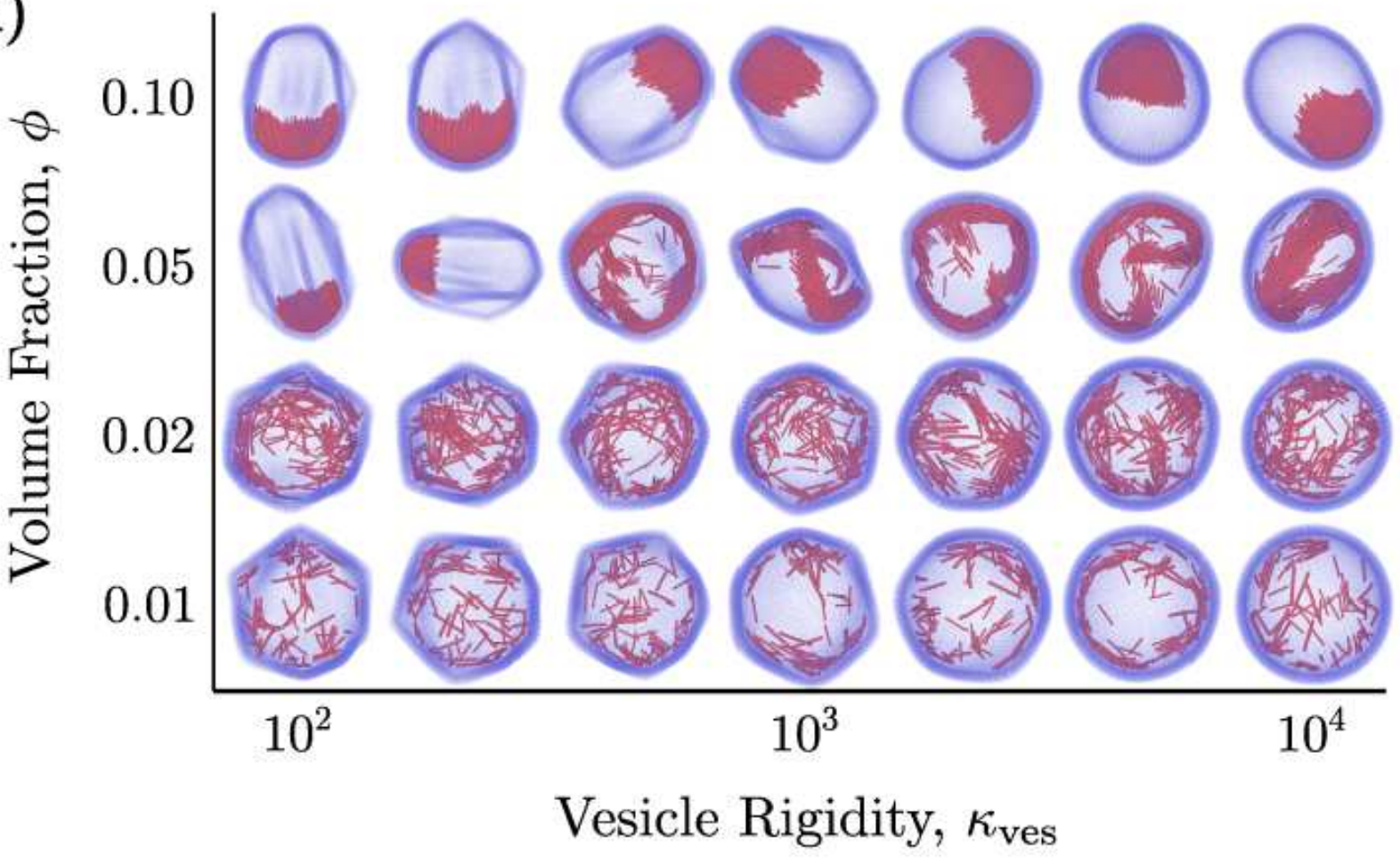

b)
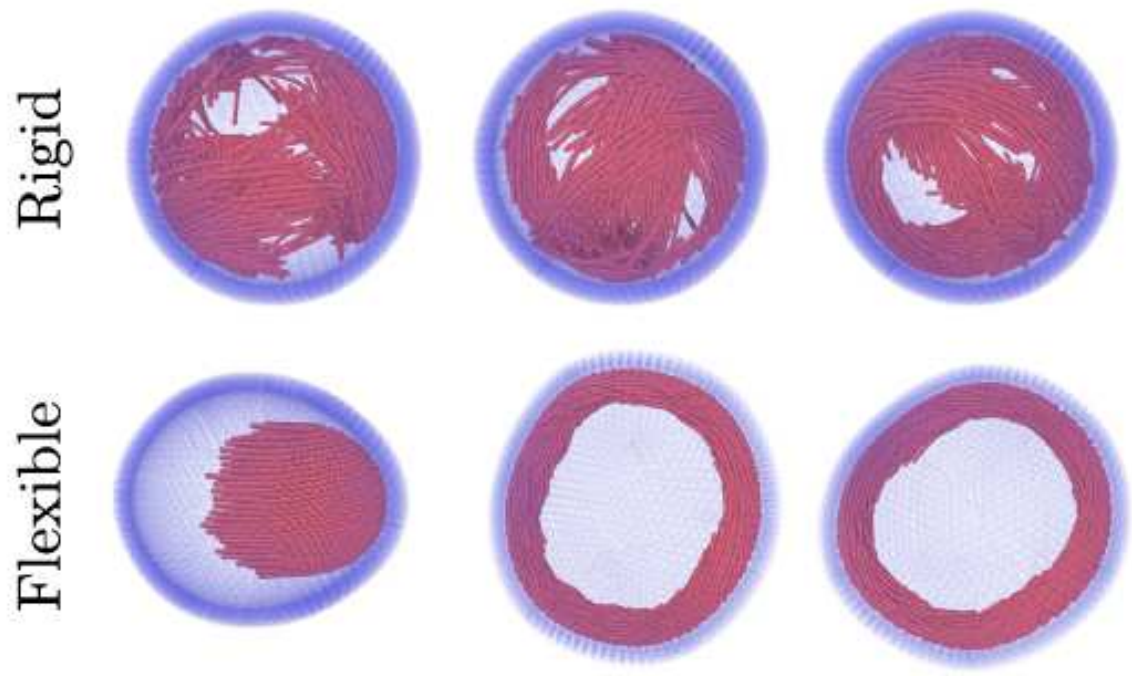

$$
a=15.5
$$

$$
a=20.5
$$$$
a=25.5
$$

Aspect Ratio

Figure 6

Please see manuscript .pdf for full caption.

\section{Supplementary Files}

This is a list of supplementary files associated with this preprint. Click to download. 
- movie1.mp4

- movie2.mp4

- movie3.mp4

- movie4.mp4

- movie5.mp4

- movie6.mp4

- supplemental.pdf 